\title{
Selection of Sustainable Redi Fishing Technology Unit in Buton Regency Southeast Sulawesi
}

\author{
Bahdad $^{1 *}$, Budi Hascaryo Iskandar ${ }^{2}$, Mulyono S. Baskoro² and M. Imron ${ }^{2}$ \\ ${ }^{1}$ Graduate School, Study Program of Marine Fisheries Technology \\ ${ }^{2}$ Staf Pengajar Lecturer of Departement of Fisheries Resource Utilization , FPIK IPB
}

${ }^{*}$ Corresponding author's email: kholis2336 [AT] gmail.com

\begin{abstract}
Environmentally friendly fishing technology of unit of redi is needed in sustainable fisheries management. The purpose of this study was to determine fishing technology unit of competitive and sustainable redi . Data collection was carried out from January to April 2018 in Buton Regency, Southeast Sulawesi Province by using survey method. The analytical data method used was scoring biological, technical and socio economic aspects the fishing technology unit of redi. Results of research showed that combined analysis of biological, technical and socioe and economic aspects have the value of the VA fishing unit redi by H.Usman (12.981) was higher than the other ten fishing equipment of redi. Thus, the fishing unit redi by H.Usman was a selected fishing technology unit of redi competitive and sustainable in Buton Regency Southeast Sulawesi Province.
\end{abstract}

Keywords----competitive , Buton District , redi fishing technology, , sustainable fishing

\section{INTRODUCTION}

The development of redi fisheries in ButonDistrict is intended to increase the number of fisheries production as an effort to pursue economic growth, but not all of this are done by taking into account the sustainability of fish resources and their habitat and also keeping them sustainable. To realize capture fisheries that are able to maintain and develop the sustainability of resource management, they must apply the elements of ecological sustainability, socioeconomic sustainability, community sustainability (Dahuri, 2007). All these aspects are very important and the interrelationshi cannot be separated in every application of technology in a region.

Sustainability, in the development of fisheries and the use of existing resources is sustainability which can simultaneously improve resource availability and environmental conditions. The concept of sustainability can actually be applied, so that the welfare of fisheries communities as resource users can be improved (Fauzi and Anna, 2005). The use of fish resources through redi fishing equipment is aimed to fullfill the needs of fishermen and on a commercial scale intended for local consumers in ButonDistrict and regional needs between provinces. Some instructions on fish resources at the study site show symptoms of overfishing, namely fishground, whose position is farther away from the beach location, and decrease in capture, as well as the smaller size of the capture and the increasing number of vessels. This phenomenon has also been investigated by Merta (1992), in the case of lemuru fish that the signs similar. Exploitation of fish resources in this area is generally dominated by redi fishing equipment, mainly from fish production obtained by local fishermen.

Several other types of fishing equipment used by Buton District fishermen to capture fish are trolling, gillnet, pole and line, charts, and others. The dominance of redi fishing equipment is due to its greater capture capacity and involves many fishermen in its operations. The technology used for captureing these fish is relatively simple, namely using FADs tools to be more efficient and economical because the intended target is clear and fuel can be predicted for use. It only needs the experience of the crew and the detection of certainty about the mob of fish to settle around FADs. So there is no need to linger around looking for fish and observing natural signs as the location of fish in a water location.

Redi in Buton District only used 1 ship in the fishing process. The ship serves to place nets, throw nets, draw ballast straps (drawstring) when the ship finsihes encircling the hordes of fish and lift the nets back, while the small boat aids are used to release the FADs from the anchorage / anchor so that the boat can easily circle fish hordes at that location. Redi is a rectangular net, which is used to capture pelagic fish in the form of schooling with a certain volume.

These fishing equipments are classified into surrounding nets. Redi fishing equipment is very effective and efficient in its operational process, especially for small pelagic fish. This is also confirmed by previous research conducted by Brant (1984) who states that redi is an effective fishing tool for captureing pelagic fish. In the process of operating redi fishing equipment, sometimes it can also get other fish resources such as large pelagic fish, squid and fish, not the target of capture. This is because of the symbiosis of the food chain. The size of the redi net in this area is generally 1 inch in size, the material is made of monofilament or better known as "string thread". On the other hand this fishing equipment is very good and effective and efficient to use but does not rule out the possibility because this dominance has resulted in unbalanced fishing with other fishing equipment such as gillnet nets and fishing rods carried out by traditional fishermen. The two horrendous cantrang cases are just one example of how fishing equipment that initially did not cause problems 
because it is still a reasonable size for both fishing equipment and ships. Along with the development of technological equipment by large capital owners, the dimensions were changed to be much longer and their fishing vessels and also the size of the net far deeper even touching the bottom of the water resulted in excessive exploitation of fish resources even though they were swept away by cantrang nets. Not to mention the impact on the environment of fish resources in these locations. Referring to this experience, it is appropriate to re-evaluate the fishing equipment besides being efficient but still adhere to the sustainability of fish resources and their habitat. The purpose of this study was to select redi fishing equipment in the area of Buton District. In order to obtain one of the best redi fishing tools that can operate sustainably and certainly beneficial in Buton District.

\section{METHODOLOGY}

\section{Data Type and Source}

The types of data taken for this study were data related to social, technical, and economic aspects of redi fishing equipmentr. The source of this research data was obtained from redi fishermen, redi owners or small business owners and from related institutions.

\section{Data Collection Method}

The method of data collection was carried out through independent interviews with redi fishermen, redi owners, and parties related to the data needs. In addition, some data related to the measurement was carried out by direct measurement with the data. The detailed explanation is presented as follows:

\section{Biological Aspect}

The parameters measured were related to things that have a social impact on redi fishing equipment, namely (1) Length of fishing operations (months), which is related to fishing operations carried out for a year, (2) The composition of the capturement which is related to the type of capture percentage value and (3) mesh size, which is related to the mesh size of their fishing net. 6) Fishing ground characteristic, which is related to water temperature in the study area.

\section{Technical Aspect}

Technical aspects included (1) The height of the ship (m), which is the size of the vessel from keel to thunderbolt, Length of the net (m), measured from the end of the net to the other end of the net (m) measured from the top of the net to the lower part, engine power (cylinder) is the engine power possessed by the ship, tonnage size (GT) which is related to the ship's loading capacity to the goods on board, the FADs dimension $(\mathrm{m})$ is related to the length and width of the FADs.

\section{Social Aspect}

The number of manpowers (ABK) is related to the use of labor involved in each fishing operation, the revenue of fishermen per year ( $\mathrm{Rp}$ ) which is related to fishermen's net revenue for a year in fishing operations, fishermen's experience relates to the years fishermen follow fishing activities , the possibility of ownership that is related to ownership, either in the form of cash or credit for purchasing the shipl, operational security, which is related to the level of safety of the ship when carrying out fishing operations.

Economic aspect of efficency criteria

Data needed for analysis of economic aspects are (1) gross revenue per year (Rp), gross receipts per trip (Rp), gross receipts per operating hour $(\mathrm{Rp})$, receipts per workforce $(\mathrm{Rp})$

Economic aspect of business investment criteria

Data needed for analysis of economic aspects are NPV (1) (Rp), Net B / C, (2) IRR (\%), and (3) Total Revenue (TR)

\section{Data Analysis Method}

Data obtained related to the interview in the next scoring is placed in the table provided. Whereas related to the calculation, the data will be included in the appropriate calculation equation.

Data Analysis

Scoring Method

The method used for the analysis of the selection of the right type of redi fishing equipment in order to obtain a packaged fishing equipment in one unit as a recommendation for the selection of equipment that represents all social, technical, social and economic aspects in Buton Regency by scoring method. This method is based on the feasibility analysis of social aspects, technical aspects, social aspects, and economic aspects (Mangkusubroto and Trisnadi 1985 referred to in Aminah 2009) as follows:

$$
v(X)=\frac{X-X_{0}}{X_{1}-X_{0}}
$$




$$
V(A)=\sum_{i=1}^{n} V_{1}\left(X_{1}\right):(i=1,2,3 \ldots \ldots n)
$$

description:
$\mathrm{V}(\mathrm{X})$
$=$ Score function of $X$ variable
$\mathrm{X}$
$=$ Score of $\mathrm{X}$ variable
$\mathrm{X} 1=$ Highest score of $\mathrm{X}$ variable
$\mathrm{XO} \quad=$ Lowest score of $\mathrm{X}$ variable
$\mathrm{V}(\mathrm{A}) \quad=\quad$ score function of alternative .
$\mathrm{V} 1(\mathrm{X} 1) \quad=$ score function from alternative of I-th criteria

This method can be used in criteria assessment which has a different unit (multidimensional)

\section{RESULTS}

Biological aspect assesment

The results of the analysis of social analysis obtained from interviews with redi fishermen and also measurements in the Buton Southeast Sulawesi regency, results were obtained as presented in Table 1.

Table 1.

Biological aspect assesment of redi ship in Buton District

\begin{tabular}{|c|c|c|c|c|c|c|c|c|c|c|}
\hline \multicolumn{4}{|c|}{ Deskripsi } & \multicolumn{7}{|c|}{ Kriteria Penilaian } \\
\hline Kapal & $x 1$ & UP1 & $\times 2$ & UP2 & $x 3$ & UP3 & $\times 4$ & UPA & $\times 5$ & UPS \\
\hline Trikol & 8 & 3 & 4 & 1 & 1 & 2 & 17 & 1 & 0,1 & 1 \\
\hline Triko 2 & 8 & 3 & 4 & 1 & 1 & 2 & 15 & 3 & 0,1 & 1 \\
\hline Triko 3 & 9 & 2 & 3 & 2 & 1 & 2 & 18 & 2 & 0,2 & 2 \\
\hline Triko 4 & 10 & 1 & 2 & 3 & 2 & 1 & 18 & 2 & 0,3 & 3 \\
\hline Triko 5 & 10 & 1 & 2 & 3 & 2 & 1 & 19 & 4 & 0,3 & 3 \\
\hline H.A. 1 & 8 & 3 & 3 & 2 & 1 & 2 & 18 & 2 & 0,1 & 1 \\
\hline H.A. 2 & 9 & 2 & 3 & 2 & 1 & 2 & 17 & 1 & 0,2 & 2 \\
\hline H.A. 3 & 9 & 2 & 2 & 3 & 2 & 1 & 15 & 3 & 0,3 & 3 \\
\hline$A C$ & 10 & 1 & 2 & 3 & 1 & 2 & 17 & 1 & 0,3 & 3 \\
\hline H.U & 9 & 2 & 3 & 2 & 2 & 1 & 18 & 2 & 0,3 & 3 \\
\hline Min $=$ & 8 & & 2 & & 1 & & 15 & & 0.1 & \\
\hline Max $=$ & 10 & & 3 & & 2 & & 19 & & 0,3 & \\
\hline
\end{tabular}

description :

X1 The lenght of fish capturement operation (month)

$\mathrm{X} 2$ composition of capturement result

X3 mesh size (inci)

X4 capturement result size $(\mathrm{cm})$

X5 By capture

X6 fishing ground charateristics

UP priority size

Assessment of biological aspects by using the value function, the Redi H.A 3 ship was on the first rank, followed by the second rank Triko 3 and the third order H.A1 shipl. The complete assessment of biological aspects is presented in Table 2 can be seen in Table 2 below. Standardization carried out on all redi capture devices that had different score attributes. 
Table 2 Scoring score and standardization of value function viewed from Biological aspect in Buton District

\begin{tabular}{|c|c|c|c|c|c|c|c|c|c|c|c|}
\hline \multirow[t]{2}{*}{ No } & \multirow[t]{2}{*}{ Kapal } & \multirow[b]{2}{*}{$\mathrm{V} 1(\mathrm{X} 1)$} & \multicolumn{3}{|c|}{ Kriteria Penilaian } & \multirow[b]{2}{*}{$\mathrm{v} 5(\times 5)$} & \multirow[b]{2}{*}{$v 6(x 6)$} & \multirow[b]{2}{*}{$v(A)$} & \multirow[b]{2}{*}{ UP } & \multirow[b]{2}{*}{$V(A)$} & \multirow[b]{2}{*}{ UP } \\
\hline & & & $\mathrm{V} 2(\mathrm{X} 2)$ & $\mathrm{V} 3(\mathrm{X} 3)$ & $\mathrm{v} 4(\mathrm{x} 4)$ & & & & & & \\
\hline & & & & & & & & & & & \\
\hline 1 & Triko 1 & 0,00 & 2 & 0 & 0,5 & 0 & 1 & 3.5 & 3 & 10 & 7 \\
\hline 2 & Triko 2 & 0,20 & 2 & 0,5 & 0 & 0 & 1 & 3,7 & 2 & 9,4 & 9 \\
\hline 3 & Triko 3 & 0,50 & 0 & 0 & 0,75 & 0,5 & 1 & 2,75 & 7 & 12,5 & 2 \\
\hline 4 & Triko 4 & 1,00 & 0 & 0 & 0,75 & 1 & 0,67 & 3,42 & 4 & 10,83 & 6 \\
\hline 5 & Triko 5 & 1,00 & 0 & 1 & 1 & 1 & 0,33 & 4,33 & 1 & 9,667 & 8 \\
\hline 6 & H.A. 1 & 0,20 & 0 & 0,5 & 0,75 & 0 & 0,67 & 2,12 & 8 & 12,23 & 3 \\
\hline 7 & H.A. 2 & 0,50 & 1 & 0 & 0,5 & 0,5 & 0,67 & 3,17 & 5 & 11,33 & 5 \\
\hline 8 & H.A. 3 & 0,10 & 0 & 0 & 0 & 1 & 0,67 & 1,77 & 9 & 12,53 & 1 \\
\hline 9 & $A C$ & 1,00 & 0 & 0 & 0,5 & 1 & 0,67 & 3,17 & 5 & 11,33 & 5 \\
\hline 10 & H.U & 0,50 & 0 & 0 & 0,75 & 1 & 0,67 & 2,92 & 6 & 11,83 & 4 \\
\hline
\end{tabular}

description :

$\mathrm{X} 1$

$\mathrm{X} 2$

$\mathrm{X} 3$

$\mathrm{X} 4$

$\mathrm{X} 5$

X6

UP

The lenght of fish capturement operation (month) composition of capturement result mesh size (inci)

capturement result size $(\mathrm{cm})$

By capture

fishing ground charateristics

priority size

\section{Technical aspect}

The factors used in this technical aspect are production per year, production per trip, production per hour of operation, production per labor, production per driving force, ship length, ship height, net length, net height, engine power (cylinder ), tonnage size, FADs dimension, length of ship. The full technical aspect assessment is presented in table 3 and table 4.

Table 3. Technical aspect assesment of redi ship in Buton Distrcit

\begin{tabular}{|c|c|c|c|c|c|c|c|c|c|c|c|c|c|c|c|}
\hline \multirow[t]{2}{*}{ No } & \multirow[t]{2}{*}{ Kapal } & \multirow[b]{2}{*}{$\mathrm{X1}$} & \multirow[b]{2}{*}{ LP1 } & \multirow[b]{2}{*}{$\times 2$} & \multicolumn{3}{|c|}{ Kriteria Penilàan } & \multirow[b]{2}{*}{$\times 4$} & \multirow[b]{2}{*}{ UP4 } & \multirow[b]{2}{*}{$x$} & \multirow[b]{2}{*}{ UPS } & \multirow[b]{2}{*}{$x_{6}$} & \multirow[b]{2}{*}{ UP6 } & \multirow[b]{2}{*}{$x 7$} & \multirow[b]{2}{*}{ UP7 } \\
\hline & & & & & UP2 & $\times 3$ & UP3 & & & & & & & & \\
\hline 1 & Triko 1 & 24 & 7 & 2 & 5 & 1,845 & 10 & 2,4 & 8 & 6 & 6 & 15,84 & 8 & $2, \pi 5$ & 8 \\
\hline 2 & Trivo 2 & 24 & 7 & 3 & 4 & 2,18 & 9 & 2,4 & 8 & 6 & 6 & 14,75 & 10 & 2,65 & 9 \\
\hline 3 & Triko 3 & 40 & 5 & 4 & 3 & 3,636 & 8 & 4 & 5 & 10 & 4 & 15 & 9 & 45 & 2 \\
\hline 4 & Trikg 4 & 56 & 4 & 5 & 2 & 5,6 & 6 & 4,667 & 4 & 9,333 & 5 & 22,2 & 2 & 42 & 4 \\
\hline 5 & Triko 5 & 96 & 1 & 6 & 1 & 9,6 & 3 & 8 & 2 & 16 & 1 & 24,5 & 1 & 48 & 1 \\
\hline 6 & HA.1 & 56 & 4 & 2 & 5 & 8 & 5 & 2,435 & 7 & 9,333 & 5 & 20,5 & 5 & 3,9 & 5 \\
\hline 7 & HA.2 & 64 & 3 & 3 & 4 & 9,143 & 4 & 3,765 & 6 & 10,67 & 3 & 21,3 & 4 & 3,45 & 7 \\
\hline 8 & HA.3 & 32 & 6 & 4 & 3 & 5,333 & 7 & 1,6 & 9 & 5,333 & 7 & 19,51 & 6 & 25 & 10 \\
\hline 9 & $A C$ & 80 & 2 & 4 & 3 & 16 & 1 & 10 & 1 & 13,33 & 2 & 17,55 & 7 & 3,7 & 6 \\
\hline 10 & $\mathrm{HU}$ & 96 & 1 & 6 & 1 & $13, \pi$ & 2 & 6.4 & 3 & 16 & 1 & 21,5 & 3 & 4,22 & 3 \\
\hline
\end{tabular}

description :

$\mathrm{X} 1 \quad$ production per year (ton)

$\mathrm{X} 2$ production per trip (ton)

X3 production per operation hour i (ton)

X4 production per manpower (ton)

X5 production per driving force

X6 ship length (m)

UP priority order 
Table 4. Technical aspect assesment of redi ship in Buton Distrcit

\begin{tabular}{|c|c|c|c|c|c|c|c|c|c|c|c|c|c|}
\hline No & Kapal & & \multicolumn{3}{|c|}{ Kriteria penilaian } & & & & & & & \\
\hline & & X8 & UP8 & X9 & UP9 & X10 & UP10 & X11 & UP11 & X12 & UP12 & X13 & UP13 \\
\hline 1 & Triko 1 & 1,5 & 8 & 300 & 5 & 47 & 2 & 4 & 2 & 12 & 6 & 1,35 & 6 \\
\hline 2 & Triko 2 & 1,7 & 5 & 300 & 5 & 47 & 2 & 4 & 2 & 12 & 6 & 1,125 & 8 \\
\hline 3 & Triko 3 & 1,35 & 0 & 450 & 4 & 68 & 1 & 4 & 2 & 17 & 4 & 1,125 & 8 \\
\hline 4 & Triko 4 & 1,5 & 8 & 450 & 4 & 68 & 1 & 6 & 1 & 17 & 4 & 1,25 & 7 \\
\hline 5 & Triko 5 & 2,4 & 2 & 600 & 2 & 68 & 1 & 6 & 1 & 25 & 2 & 1,6 & 5 \\
\hline 6 & H.A.1 & 1,95 & 3 & 500 & 3 & 68 & 1 & 6 & 1 & 20 & 3 & 1,6 & 5 \\
\hline 7 & H.A.2 & 1,56 & 7 & 450 & 4 & 68 & 1 & 6 & 1 & 17 & 4 & 2,24 & 4 \\
\hline 8 & H.A.3 & 1,75 & 4 & 250 & 6 & 68 & 1 & 4 & 2 & 15 & 5 & 2,8 & 3 \\
\hline 9 & AC & 1,65 & 6 & 450 & 4 & 45 & 3 & 6 & 1 & 17 & 4 & 4,2 & 1 \\
\hline 10 & H.U & 2,7 & 1 & 650 & 1 & 68 & 1 & 6 & 1 & 30 & 1 & 3 & 2 \\
\hline
\end{tabular}

description :

$\begin{array}{ll}\text { X8 } & \text { Ship height }(\mathrm{m}) \\ \text { X9 } & \text { net lenght }(\mathrm{m}) \\ \text { X10 } & \text { net height }(\mathrm{m}) \\ \text { X11 } & \text { machine power (cylinder) } \\ \text { X12 } & \text { tonage size }(\mathrm{GT}) \\ \text { X13 } & \text { FADs dimension (m) } \\ \text { X14 } & \text { ship lenght }(\mathrm{m}) \\ \text { UP } & \text { priority order }\end{array}$

Assesment of technical aspect using value function, found that H.U redi ship ranked first, followed by the redi triko 5, the third order redi AC. Triko 4 and H.A 2 were in fourth and fifth. The full assessment of technical aspects is presented in Tables 3 and 4. Standardization carried out on all redi capture equipment that has different score attribute.

Table 5 Scoring score and standarization viewed from technical aspect in Buton District

\begin{tabular}{|l|l|l|l|l|l|l|l|l|l|l|l|l|l|l|l|l|l|}
\hline \multicolumn{10}{|c|}{ No Kapal } & \multicolumn{10}{|c|}{ Kriteria Penilaian } \\
\hline & & $V(X 1)$ & $V(X 2)$ & $V(X 3)$ & $V(X 4)$ & $V(X 5)$ & $V(X 6)$ & $V(X 7)$ & $V(X 8)$ & $V(X 9)$ & $V(X 10)$ & $V(X 11)$ & $V(X 12)$ & $V(X 13)$ & $V(A)$ & $U P$ \\
\hline 1 & Triko 1 & 0,000 & 0,000 & 0,000 & 0,261 & 0,083 & 0,112 & 0,109 & 0,111 & 0,125 & 0,087 & 0,087 & 0,000 & 0,000 & 0,975 & 10 \\
\hline 2 & Triko 2 & 0,000 & 0,250 & 0,028 & 0,261 & 0,083 & 0,000 & 0,065 & 0,259 & 0,125 & 0,087 & 0,087 & 0,000 & 0,079 & 1,325 & 9 \\
\hline 3 & Triko 3 & 0,222 & 0,500 & 0,151 & 0,783 & 0,583 & 0,026 & 0,870 & 0,000 & 0,500 & 1,000 & 1,000 & 0,278 & 0,079 & 5,991 & 7 \\
\hline 4 & Trik0 4 & 0,444 & 0,750 & 0,316 & 1,000 & 0,500 & 0,764 & 0,739 & 0,111 & 0,500 & 1,000 & 1,000 & 0,278 & 0,035 & 7,438 & 4 \\
\hline 5 & Triko 5 & 1,000 & 1,000 & 0,653 & 2,087 & 1,333 & 1,000 & 1,000 & 0,778 & 0,875 & 1,000 & 1,000 & 0,722 & 0,088 & 12,536 & 2 \\
\hline 6 & H.A.1 & 0,556 & 0,000 & 0,519 & 0,272 & 0,500 & 0,590 & 0,609 & 0,444 & 0,625 & 1,000 & 1,000 & 0,444 & 0,088 & 6,646 & 6 \\
\hline 7 & H.A.2 & 0,556 & 0,250 & 0,615 & 0,706 & 0,667 & 0,672 & 0,413 & 0,156 & 0,500 & 1,000 & 1,000 & 0,278 & 0,312 & 7,123 & 5 \\
\hline 8 & H.A.3 & 0,111 & 0,500 & 0,294 & 0,000 & 0,000 & 0,488 & 0,000 & 0,296 & 0,000 & 1,000 & 1,000 & 0,167 & 0,509 & 4,365 & 8 \\
\hline 9 & A.C & 0,778 & 0,500 & 1,193 & 2,739 & 1,000 & 0,287 & 0,522 & 0,222 & 0,500 & 0,000 & 0,000 & 0,278 & 1,000 & 9,018 & 3 \\
\hline 10 & H.U & 1,000 & 1,000 & 1,000 & 1,565 & 1,333 & 0,692 & 0,748 & 1,000 & 1,000 & 1,000 & 1,000 & 1,000 & 0,579 & 12,918 & 1 \\
\hline
\end{tabular}

\section{Social Aspect Assesment}

The results of the social analysis obtained from interviews with redi fishermen and also measurements in Buton District, Southeast sulawesi, the results obtained as presented in table 6.

Table 6. Technical aspect assesment of redi fishing equipment in Buton Distrcit

\begin{tabular}{|c|c|c|c|c|c|c|c|c|c|}
\hline \multirow[b]{2}{*}{ No } & \multirow[t]{2}{*}{ Deskripsi } & \multirow[b]{2}{*}{$\mathrm{X} 1$} & \multirow[b]{2}{*}{ UP1 } & \multirow[b]{2}{*}{$\mathrm{X} 2$} & \multirow[b]{2}{*}{ UP2 } & \multicolumn{2}{|c|}{ Kriteria Penilaian } & \multirow[b]{2}{*}{$\mathrm{X} 4$} & \multirow[b]{2}{*}{ UP4 } \\
\hline & & & & & & X3 & UP3 & & \\
\hline & Kapal & & & (jt) & & & & & \\
\hline & & & & & & & & & \\
\hline 1 & Triko 1 & 10 & 2 & 16 & 4 & 2 & 7 & 10 & 3 \\
\hline 2 & Triko 2 & 10 & 2 & 20 & 6 & 3 & 6 & 7 & 4 \\
\hline 3 & Triko 3 & 10 & 2 & 21,6 & 3 & 10 & 3 & 7 & 4 \\
\hline 4 & Triko 4 & 12 & 3 & 21,6 & 3 & 5 & 5 & 10 & 3 \\
\hline 5 & Triko 5 & 12 & 3 & 21,6 & 3 & 7 & 4 & 10 & 3 \\
\hline 6 & H.A. 1 & 23 & 7 & 16 & 4 & 20 & 1 & 20 & 1 \\
\hline 7 & H.A. 2 & 17 & 5 & 16 & 4 & 10 & 3 & 15 & 2 \\
\hline 8 & H.A. 3 & 20 & 6 & 17 & 5 & 15 & 2 & 10 & 3 \\
\hline 9 & $\mathrm{AC}$ & 8 & 1 & 45 & 1 & 15 & 2 & 5 & 5 \\
\hline 10 & H.U & 15 & 4 & 35 & 2 & 7 & 4 & 5 & 5 \\
\hline
\end{tabular}


description :

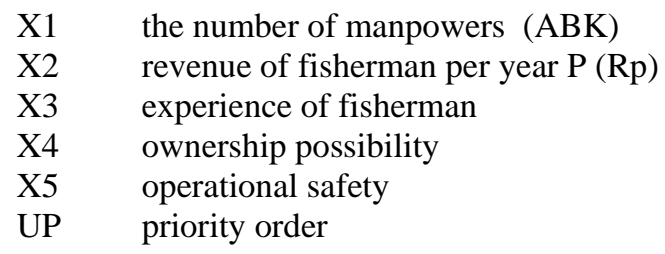

Based on the calculation of the scoring results and standardization of social aspects, then for Buton District, the redi HA 1 fishing equipement was on the first rank, followed by the Redi H.A2 fishing equipment while the third rank redi fishing equipment was occupied by four redi fishing equipment, each of them was Triko 1 ,

Table 7 Standardization of redi capturement social aspect in Buton distrcit

\begin{tabular}{|c|c|c|c|c|c|}
\hline \multirow[b]{2}{*}{ No } & \multirow[b]{2}{*}{ Kapal } & \multicolumn{2}{|c|}{ Aspek Penilaian } & \multirow[b]{2}{*}{ V3(X3) } & \multirow[b]{2}{*}{$\mathrm{V} 4(\times 42)$} \\
\hline & & V1(X1) & $\sqrt{ } 2(\times 2)$ & & \\
\hline 1,00 & Triko 1 & 0,13 & 0,00 & 0,00 & 0,33 \\
\hline 2,00 & Triko 2 & 0,13 & 0,14 & 0,06 & 0,13 \\
\hline 3,00 & Triko 3 & 0,13 & 0,19 & 0,44 & 0,13 \\
\hline 4,00 & Triko 4 & 0,27 & 0,19 & 0,17 & 0,33 \\
\hline 5,00 & Triko 5 & 0,27 & 0,19 & 0,28 & 0,33 \\
\hline 6,00 & H.A. 1 & 1,00 & 0,00 & 1,00 & 1,00 \\
\hline 7,00 & H.A. 2 & 0,60 & 0,00 & 0,44 & 0,67 \\
\hline 8,00 & H.A. 3 & 0,80 & 0,03 & 0,72 & 0,33 \\
\hline 9,00 & $A C$ & 0,00 & 1,00 & 0,72 & 0,00 \\
\hline \multirow[t]{3}{*}{10,00} & H.U & 0,47 & 0,66 & 0,28 & 0,00 \\
\hline & Min & 8,00 & 16,00 & 2,00 & 5,00 \\
\hline & Max & 23,00 & 45,00 & 20,00 & 20,00 \\
\hline
\end{tabular}

\section{Economic aspect assesment of business effiency of redi fishing business.}

The results of economic aspects assesment of the business efficiency criteria for redi fishing obtained from interviews with redi fishermen and also measurements in the Buton District of Southeast Sulawesi, obtained results as presented in the Table 8

Table 8. Economic assesment of efficiency criteria of redi fishing business

\begin{tabular}{|c|c|c|c|c|c|c|c|c|c|}
\hline No & Kapal & & & & & Kriteria Penilaian & & & \\
\hline & & $\mathrm{X}_{1}$ & UP1 & $\mathrm{X} 2$ & UP2 & X3 & UP3 & $\mathrm{X} 4$ & UP4 \\
\hline 1 & Triko 1 & 360,000 & 7 & $45.000 .000,000$ & 7,000 & $5.000 .000,000$ & 9,000 & $4.500 .000,000$ & 8 \\
\hline 2 & Triko 2 & 360,000 & 7 & $45.000 .000,000$ & 7,000 & $5.000 .000,000$ & 9,000 & $4.500 .000,000$ & 8 \\
\hline 3 & Triko 3 & 600,000 & 5 & $75.000 .000,000$ & 5,000 & $8.333 .333,333$ & 1,000 & $7.500 .000,000$ & 5 \\
\hline 4 & Triko 4 & 840,000 & 4 & $105.000 .000,000$ & 4,000 & $10.500 .000,000$ & 7,000 & $8.750 .000,000$ & 4 \\
\hline 5 & Triko 5 & 1440,000 & 1 & $180.000 .000,000$ & 1,000 & $16.363 .636,364$ & 4,000 & $15.000 .000,000$ & 2 \\
\hline 6 & H.A. 1 & 840,000 & 4 & $105.000 .000,000$ & 4,000 & $15.000 .000,000$ & 6,000 & $4.565 .217,391$ & 7 \\
\hline 7 & H.A. 2 & 960,000 & 3 & $120.000 .000,000$ & 3,000 & $17.142 .857,143$ & 3,000 & $7.058 .823,529$ & 6 \\
\hline 8 & H.A. 3 & 540,000 & 6 & $60.000 .000,000$ & 6,000 & $10.000 .000,000$ & 8,000 & $3.000 .000,000$ & 9 \\
\hline 9 & $\mathrm{AC}$ & $1.200,000$ & 2 & $150.000 .000,000$ & 2,000 & $25.000 .000,000$ & 5,000 & $18.750 .000,000$ & 1 \\
\hline 10 & H.U & 1440,000 & 1 & $180.000 .000,000$ & 1,000 & $25.714 .285,714$ & 3,000 & $12000.000,000$ & 3 \\
\hline
\end{tabular}

description :

$\mathrm{X} 1 \quad$ gross revenue per year $(\mathrm{Rp})$

$\mathrm{X} 2$ gross revenue per trip (Rp)

$\mathrm{X} 3$ gross revenue per operation hour (Rp)

$\mathrm{X} 4 \quad$ revenue per manpower $(\mathrm{Rp})$

UP priority order

Economic aspect assesment of business efficiency criteria for redi fishing by using the value function, the redi HU ship was on the first rank, the redi AC ship on the second rank and the third was occupied by redi triko 5 fishing equipment. The other rankings are presented in the table 9 below. 


\begin{tabular}{|c|c|c|c|c|c|c|c|}
\hline \multirow[t]{2}{*}{ No } & \multirow[t]{2}{*}{ Kapal } & \multirow[b]{2}{*}{$\mathrm{V}(\mathrm{X} 1)$} & \multicolumn{3}{|c|}{ Kriteria Penilaian } & \multirow[t]{2}{*}{$V(A)$} & \multirow[t]{2}{*}{ UP } \\
\hline & & & $\mathrm{V}(\mathrm{X} 2)$ & $\mathrm{V}(\mathrm{X} 3)$ & $\mathrm{V}(\mathrm{X} 4)$ & & \\
\hline 1 & Triko 1 & 0,000 & 0,000 & 0,000 & 0,095 & 0,095 & 9 \\
\hline 2 & Triko 2 & 0,000 & 0,000 & 0,000 & 0,095 & 0,095 & 9 \\
\hline 3 & Triko 3 & 0,222 & 0,222 & 0,161 & 0,286 & 0,891 & 7 \\
\hline 4 & Triko 4 & 1,000 & 0,444 & 0,266 & 0,365 & 2,075 & 4 \\
\hline 5 & Triko 5 & 1,000 & 1,000 & 0,549 & 0,762 & 3,310 & 3 \\
\hline 6 & H.A. 1 & 0,444 & 0,444 & 0,483 & 0,099 & 1,471 & 6 \\
\hline 7 & H.A. 2 & 0,556 & 0,556 & 0,586 & 0,258 & 1,955 & 5 \\
\hline 8 & H.A. 3 & 0,167 & 0,111 & 0,241 & 0,000 & 0,519 & 8 \\
\hline 9 & $\mathrm{AC}$ & 0,778 & 0,778 & 0,966 & 1,000 & 3,521 & 2 \\
\hline 10 & H.U & 1,000 & 1,000 & 1,000 & 0,571 & 3,571 & 1 \\
\hline
\end{tabular}

\section{Economic assesment of business investment of redi fishing}

The results of the economic aspect assesment of the investment criteria for the redi fishing effort obtained from interviews with redi fishermen and also measurements in the Buton District, Southeast Sulawesi, the results obtained as presented in table 9

\begin{tabular}{|c|c|c|c|c|c|c|c|c|c|}
\hline \multirow{2}{*}{$\begin{array}{l}\text { Table } \\
\text { No } \\
\end{array}$} & 9. & economic & \multicolumn{2}{|c|}{ assesment } & aspect & \multicolumn{2}{|c|}{ business } & vestment & \multirow[t]{2}{*}{ criteria } \\
\hline & Kapal & & & & & eria Penil & & & \\
\hline & & $\mathrm{X} 1$ & UP1 & $\mathrm{X} 2$ & UP2 & X3 & UP3 & $\mathrm{X} 4$ & UP4 \\
\hline 1 & Triko 1 & 780000000 & 5 & 1,610234 & 4 & 69,9641 & 5 & 1,429304 & 6 \\
\hline 2 & Triko 2 & 783500000 & 6 & 1,816396 & 3 & 165,2128 & 3 & 0,60528 & 3 \\
\hline 3 & Triko 3 & 815500000 & 7 & 1,880816 & 2 & 275,6488 & 2 & 0,36278 & 2 \\
\hline 4 & Triko 4 & 965500000 & 8 & 1,894533 & 1 & 322,7646 & 1 & 0,309823 & 1 \\
\hline 5 & Triko 5 & 1120000000 & 9 & 1,578529 & 6 & 47,12129 & 7 & 2,122183 & 7 \\
\hline 6 & H.A. 1 & 608000000 & 2 & 1,4462 & 8 & 42,62623 & 9 & 2,345973 & 9 \\
\hline 7 & H.A. 2 & 616000000 & 3 & 1,568192 & 7 & 56,46591 & 6 & 1,77098 & 5 \\
\hline 8 & H.A. 3 & 716000000 & 4 & 1,261292 & 9 & 15,62395 & 10 & 6,400429 & 10 \\
\hline 9 & $A C$ & 530000000 & 1 & 1,590086 & 5 & 84,0234 & 4 & 1,190145 & 4 \\
\hline 10 & H.U & 1152750000 & 10 & 1,591866 & 5 & 46,44559 & 8 & 2,153057 & 8 \\
\hline
\end{tabular}

description :

$\begin{array}{ll}\text { X1 } & \text { NPV }(\mathrm{Rp}) \\ \text { X2 } & \text { Net B/C } \\ \text { X3 } & \text { IRR }(\%) \\ \text { X4 } & \text { total revenue (TR) } \\ \text { UP } & \text { priority order }\end{array}$

Assessment of the economic aspects of the investment criteria for the redi fishing business obtained from interviews with redi fishermen and also measurements in the Buton District, Southeast Sulawsi , the results obtained as presented in table 9 
table 10 Standarization of economic aspect of investment criteria of redi fishing equipment.

\begin{tabular}{|c|c|c|c|c|c|c|c|}
\hline \multirow[t]{2}{*}{ No } & \multirow[t]{2}{*}{ Kapal } & \multirow[b]{2}{*}{$\mathrm{V}(\mathrm{X} 1)$} & \multicolumn{3}{|c|}{ Kriteria Penilaian } & \multirow[b]{2}{*}{$V(A)$} & \multirow[b]{2}{*}{ UP } \\
\hline & & & $\mathrm{V}(\mathrm{X} 2)$ & $\mathrm{V}(\mathrm{X} 3)$ & $\mathrm{V}(\mathrm{X} 4)$ & & \\
\hline 1 & Triko 1 & 0,401445 & 0,633242 & 0,176923 & 0,183805 & 1,395414 & 6 \\
\hline 2 & Triko 2 & 0,407065 & 0,633242 & 0,487037 & 0,04851 & 1,575854 & 5 \\
\hline 3 & Triko 3 & 0,45845 & 0,633242 & 0,846599 & 0,008695 & 1,946985 & 3 \\
\hline 4 & Triko 4 & 0,699318 & 0,633242 & 1 & 0 & 2,332559 & 1 \\
\hline 5 & Triko 5 & 0,947411 & 0,633242 & 0,10255 & 0,297566 & 1,980769 & 2 \\
\hline 6 & H.A. 1 & 0,125251 & 0,633242 & 0,087915 & 0,33431 & 1,180717 & 7 \\
\hline 7 & H.A. 2 & 0,138097 & 0,633242 & 0,132975 & 0,239903 & 1,144217 & 8 \\
\hline 8 & H.A. 3 & 0,298675 & 0,633242 & 0 & 1 & 1,931917 & 4 \\
\hline 9 & $\mathrm{AC}$ & 0 & 0,633242 & 0,222697 & 0,144538 & 1,000477 & 10 \\
\hline 10 & H.U & 1 & 0,633242 & 0,10035 & $-0,69736$ & 1,036227 & 9 \\
\hline
\end{tabular}

\section{Combination aspect assesment ( biological, technical,social, economic aspect)}

The selection of the right redi fishing equipment in determining the best alternative, environmentally friendly and sustainable technology package in Buton District, Southeast Sulawesi, requires a joint analysis, which is a combination of biological, technical, social and economic analysis. The combined analysis function for determining redi fishing equipment that is feasible to use and can be developed in the future. The results of the analysis showed that Buton District redi fishing equipment that was suitable for use was redi fishing equipment owned by Triko 4 company so that fishing equiment was given the highest priority for the selection of appropriate redi fishing equipment in determining the best alternative, environmentally friendly and sustainable technology package in Buton District, Southeast Sulawesi (table 13).

Table 12. Penilaian aspek biologi, teknis, sosial dan ekonomi penangkapan redi Kabupaten Buton

\begin{tabular}{|c|c|c|c|c|c|c|c|c|c|c|c|}
\hline \multirow[t]{2}{*}{ No } & \multirow[t]{2}{*}{ Kapal } & \multirow[b]{2}{*}{$\mathrm{X} 1$} & \multirow[b]{2}{*}{ UP1 } & \multirow[b]{2}{*}{$\mathrm{X} 2$} & \multirow[b]{2}{*}{ UP2 } & \multicolumn{2}{|c|}{ Kriteria Penilaian } & \multirow[b]{2}{*}{$\mathrm{X} 4$} & \multirow[b]{2}{*}{ UP4 } & \multirow[b]{2}{*}{$\times 5$} & \multirow[b]{2}{*}{ UP5 } \\
\hline & & & & & & $\mathrm{X} 3$ & UP3 & & & & \\
\hline 1 & Triko 1 & 10,000 & 7,000 & 0,975 & 10,000 & 0,467 & 9,000 & 0,095 & 9,000 & 1,395 & 6 \\
\hline 2 & Triko 2 & 9,400 & 9,000 & 1,167 & 9,000 & 0,460 & 10,000 & 0,095 & 9,000 & 1,576 & 5 \\
\hline 3 & Triko 3 & 12,500 & 2,000 & 5,833 & 7,000 & 0,904 & 8,000 & 0,891 & 7,000 & 1,947 & 3 \\
\hline 4 & Triko 4 & 10,833 & 6,000 & 7,368 & 4,000 & 0,960 & 7,000 & 2,075 & 4,000 & 2,333 & 1 \\
\hline 5 & Triko 5 & 9,667 & 8,000 & 12,536 & 2,000 & 1,071 & 6,000 & 3,310 & 3,000 & 1,981 & 2 \\
\hline 6 & H.A. 1 & 12,233 & 3,000 & 6,646 & 6,000 & 3,000 & 1,000 & 1,471 & 6,000 & 1,181 & 7 \\
\hline 7 & H.A. 2 & 11,333 & 5,000 & 7,123 & 5,000 & 1,711 & 4,000 & 1,955 & 5,000 & 1,144 & 8 \\
\hline 8 & H.A. 3 & 12,533 & 1,000 & 4,365 & 8,000 & 1,890 & 2,000 & 0,519 & 8,000 & 1,932 & 4 \\
\hline 9 & $A C$ & 11,333 & 5,000 & 9,018 & 3,000 & 1,722 & 3,000 & 3,521 & 2,000 & 1,000 & 10 \\
\hline 10 & H.U & 11,833 & 4,000 & 12,918 & 1,000 & 1,400 & 5,000 & 3,571 & 1,000 & 1,036 & 9 \\
\hline
\end{tabular}

description :
X1 Biological aspect
X2 Technical aspect
X3 Social aspect
X4 Economic aspect of redi business efficeincy criteria
X5 economic aspect of redi businessinvestment criteria
UP priority order

Table 13. (Standardization of biological, technical, social and economic aspect of redi fishing in Buton District)

\begin{tabular}{|c|c|c|c|c|c|c|c|c|}
\hline No & Kapal & \multicolumn{3}{|c|}{ Kriteria Penilaian } & & & \\
\hline & & $\mathrm{V}(\mathrm{X} 1)$ & $\mathrm{V}(\mathrm{X} 2)$ & $\mathrm{V}(\mathrm{X} 3)$ & $\mathrm{V}(\mathrm{X} 4)$ & $\mathrm{V}(\mathrm{X} 5)$ & $\mathrm{V}(\mathrm{A})$ & $\mathrm{UP}$ \\
\hline 1 & Triko 1 & 0,191 & 0,000 & 0,003 & 0,000 & 0,297 & 0,491 & 9 \\
\hline 2 & Triko 2 & 0,000 & 0,016 & 0,000 & 0,000 & 0,432 & 0,448 & 10 \\
\hline 3 & Triko 3 & 0,989 & 0,407 & 0,175 & 0,229 & 0,711 & 2,511 & 7 \\
\hline 4 & Triko 4 & 0,457 & 0,535 & 0,197 & 0,570 & 1,000 & 2,759 & 5 \\
\hline 5 & Triko 5 & 0,085 & 0,968 & 0,241 & 0,925 & 0,736 & 2,955 & 2 \\
\hline 6 & H.A. 1 & 0,904 & 0,475 & 1,000 & 0,396 & 0,136 & 2,911 & 3 \\
\hline 7 & H.A. 2 & 0,617 & 0,515 & 0,493 & 0,535 & 0,108 & 2,268 & 8 \\
\hline 8 & H.A. 3 & 1,000 & 0,284 & 0,563 & 0,122 & 0,700 & 2,668 & 6 \\
\hline 9 & AC & 0,617 & 0,673 & 0,497 & 0,986 & 0,000 & 2,773 & 4 \\
\hline 10 & H.U & 0,777 & 1,000 & 0,370 & 1,000 & 0,027 & 3,174 & 1 \\
\hline
\end{tabular}

description :

X1 standardization result of biological aspect

X2 standardization result of technical aspect

X3 standardizationof social aspect

X4 standardization result of economic aspect efficiency criteria 
X5 standardization result of economic aspect efficiency criteria of redi business

UP priority order

\section{DISUCSSION}

Berdasarkan hasil analisis yang dilakukan dengan metode skoring terhadap aspek biologi, teknik, sosial, ekonomi, maka alat tangkap redi H.U menempati peringkat pertama untuk dikembangkan di Kabupaten Buton Sulawesi Tenggara.

\section{Biological Aspect Review}

Assessment of biological aspects is based on attributes (1) length of fishing operations (months); (2) capture composition; (3) mesh size (inches); (4) capture size ( $\mathrm{cm})$; (5) by capture; (6) characteristics of fishing ground. By using the value function, it is found that redi triko 5 ranked 1 , followed by second order triko and third order triko. Complete assessment of biological aspects is presented in Table 2 below. Standardization carried out on all redi capture devices all have different value attributes. Furthermore, the results of the analysis carried out by the scoring method on biological aspect in redi H.A. ship with the best priority for future development. Redi Triko 5 ships had a high capture operation duration as well as 4 tricycle boats and AC ship.

Placement of priority ship sequence based on standardization of value functions of biological aspects of attributes. Based on the standardization of the value function, it was found that redi triko 5 was in the first priority with a function of value $\mathrm{V}(\mathrm{A}=(4.33)$. It was based on the number of units of biological aspect value function with 4 units of function value which gave the highest point of one unit on each indicator that is the indicator of the length of time of fishing operations, the mesh size of the net used for captureing the fish, the size of the fish caught and the value of capture of wasted fish caught in. Low function value in the two biological attributes was not again gave effect to the total rating value owned by the ship in Triko 5 .

The second priority in the aspect of biological assessment of all tangka redi equipment was occupied by Triko 2 . It is based on the largest function unit value owned by the biological attributes of the capture composition, mesh size of the net, characteristics of fishing ground. One attribute was low and the other two attributes were the lowest. The third priority was redi triko 1 ship. The third rank placement is based on the function unit of the largest biological attribute value found in this fishing equipment, namely the attributes of the capture composition, the size of the capture, the characteristics of the fishing ground. The other 3 attributes were the length of time of fishing operations, the size of the fish caught and the value of capture of the caught fish was wasted with the lowest value. If we connect the size of the flying fish caught in this area, according to some sources of citations, flying fish is an important resource of small pelagic fish in Indonesian waters. In the study of Nontji (2007), it was found that fly fish in Indonesian waters consisted of five types, namely D. single, D. ruselli, D. macrosoma, D. kuroides and D. maruadsi.

The characteristics of these fish usually live in groups (schooling) with other small pelagic fish as examined by Chan et al., (1997). Flying fish tend to cluster near surface layers at a depth of 3-20 m (Amin et al., 1998). Based on one of these characteristics, the design of redi fishing equipment was designed by the inventor. The distribution starts from Java Sea, Makasar Strait, Ambon, Ternate (Eastern Indonesia). While the existence of flying fish in the Indian Ocean area can be classified in 8 types of flying fish including D. macarellus, D. sactaehelenae, D. punctatus. The existence of flying fish in the Java Sea enters the Makassar Strait and the Flores Sea while in the West season from the Indian Ocean and South China Sea. In waters around Southeast Sulawesi , flying fish is one of the main components in captureing trawler fishermen and gill net using FADs as fish collectors.

According to Widodo (2000), trawl rings and gill nets, payang and boat charts were very effective in fishing for flying fish. Durand in 1998 stated that purse seine fishing equipment contributed to the largest fly fish capture, which was 92\% compared to other fishing gears. While Gafa et al., (2003), stated that in the waters of the Sulawesi Sea, flying fish had been exploited intensively and have been overfished. According to the statistics of flying fish in Indonesian waters in 2006-2010, the capture data had increased throughout the year with an average increase of 3.68\% per year. (Ministry of Maritime Affairs and Fisheries, 2011).

According to local fishermen, fly fish are caught throughout the year. Peak season capturement occurs in AugustDecember that is signed by the high production of flying fish both in number and type compared to other months. In the Java Sea the peak of flying fish production occurs 2 times a year, namely January-March (end of the western season) and July-September (east season) Sreenivasa (1997). Research conducted by Atmaja et al. (2003) stated that flying fish of size $12 \mathrm{~cm}$ were found in the Java Sea in June. July-October found flying fish measuring $8 \mathrm{~cm}$ and in April-May found flying fish eggs and larvae. The peak season for fly fishing in Buton Regency occured in August-November (east wind season), medium season in December-March and less season in April-June. Usually flying fish that was found in larger peak seasons like D.ruselli can reach $28 \mathrm{~cm}$ long, D. macrosomas can reach $23 \mathrm{~cm}$ long and D. singles can reach $27 \mathrm{~cm}$ long.

According to LIPI Center for Research on Oceanology ((1998), generally D. macrosomas can reached $40 \mathrm{~cm}$ in length, generally $25 \mathrm{~cm}$, D. ruselli reached $40 \mathrm{~cm}$ in length, generally $20-25 \mathrm{~cm}$. Furthermore, Nontji (2002), said that in the east wind season, namely in June-September the migration pattern of flying fish into the Java Sea from the Makassar Strait and Flores Sea follows the current pattern and salinity distribution pattern. It was alleged that the pattern of the east 
season currents carried flying fish into the waters of Buton Regency. Widodo (2000), that in addition to the peak season the number of flying fishes is caught both in number and species or not caught at all.

Najamuddin et al. (2004) stated that D. ruselli fish was usually clustered with other types of flying fish in large numbers on the surface of the waters and occasionally D. Ruselli's fish entered to deeper waters with D. Lajang fish following the movement of plankton. Widjojo (2002) found that plankton was the main food of D.ruselli fish. Aprilianti, (2000) said that there was a tendency for D. ruselli at night to tend to deeper layers of water and during the day to rise to the surface layer. Widodo (2000), stated that D. Lajang fish tend to inhabit deep waters. To illustrate the maturity level of flying fish at that size, a comparison can be used from the results of the study of Najamuddin et al. (2004), D. The macrosoma of the female first matured the gonad at a length of 19.8-20.3 cm, while the male flying fish first gonads at $19.6-20.1 \mathrm{~cm}$.

According to Prihartini (2006), D. macrosoma had reached gonadal maturity at a weight of $30.5 \mathrm{~g}$, so the results of studies captured during the study have weighed above that size. Faizu (2001), from the results of his research in the Siompu waters of Buton district that flying species D. ruselli had first matured gonads at length 28.1-29.2 cm (Hariati et al., 2005), gonads first matured at length $16 \mathrm{~cm}$. Furthermore, Aprilianti (2000), the first male D. ruselli fish gonads at a size of $15.8 \mathrm{~cm}$ long and D. ruselli gonad mature women at a length of $15.3 \mathrm{~cm}$.

Report of LIPI Center for Research on Oceanology (1999), D. Ruselli could reach $30 \mathrm{~cm}$ and generally 20-25 $\mathrm{cm}$. When referring to the results of the study it can be said that most $(<85 \%)$ of D. ruselli caught have long gonads matured for the first time, but caught fish was far from their maximum length. According to Prihartini (2006), D. Ruselli had reached mature gonads at a weight of 38 grams, so the sample D. Ruselli had reached more than that size. Based on several studies, it has been shown that the size of fish caught in a puresein fishing equipment is closely related to the length and width of the fishing gear, and the size of the mesh.

The length and width of the fishing equipment are closely related to the number of obtained target fish. While the size of the net is related to the selection of the size of obtained fish . Previous research on this association had been observed by Merta (1992) and Nontji (2007) on four lengths of lemuru fish, there were sempenit (10-12.5 cm), protolan $(13-14.5 \mathrm{~cm})$, lemuru $(15-17,5 \mathrm{~cm})$ and lemuru kucing $(17.9-19 \mathrm{~cm})$. The results of the study also showed that the length and width of the fishing treatment, and the size of the net can affect the capture.

Based on the results of interviews with respondents (fishermen purse seine), the size of the captured finite ranges from 10.00 to $12.00 \mathrm{~cm}$, protolan with a length of $12.00-15.00 \mathrm{~cm}$, lemuru with a length of $15.00-17.00 \mathrm{~cm}$, and lemuru kucing with a length of $17.50-20.00 \mathrm{~cm}$. In addition to the dependence on the size of the fish caught on the size of the fishing gear and even the size dependence of the ship on fishing equipment. Even the determination of the size of fishing treatment also has a close relation to the size of the ship that will be owned. This has been required by FAO that the size of the fishing equipment must not exceed the size of 15 times of the length of the ship.

\section{Redi fishing equipment viewd from technical aspect}

The factors used in this technical aspect were production per year, production per trip, production per hour of operation, production per workforce, production per driving force, ship length, ship width (m), ship height, net length, net height, engine power (cylinder), tonnage size, FADs dimension. Evaluation of the full technical aspects is presented in table 3 and table 4.m Placement of priority sequences of ships based on standardized value functions of technical aspect attributes mentioned above.

Based on the standardization of the value function, it was found that the redi HU ship ranked on first priority with the function value $\mathrm{V}(\mathrm{A})=(12,918)$. This is based on the number of function units of the technical aspects consisting of 8 units of value functions that give the highest point on each technical attribute, namely production per year, production per trip, production per driving force, ship height, net length, net height, engine power, ship tonnage size. There are 4 other attributes that have a value in the second order. This illustrates that technical aspects of this fishing equipment was very productive and efficient. The second priority in the aspect of technical assessment of all tangka redi tools was occupied by triko 5 with $\mathrm{V}(\mathrm{A})=12,539$. It is based on the largest function unit value it has, namely on the technical attributes, namely production per year, production per trip, ship length, ship width, net height, engine power. The rest of the attributes have the position of the second value of each indicator. The third priority is the A.C redi ship. Third rank placement was based on the function units of the largest technical attribute values that exist in this fishing equipment, namely production attributes per trip, production per hour of operation, FADs dimensions. This ship had a larger production compared to other ships. In addition, this ship also had a much wider size compared to other ships. Likewise with production per manpower provided greater production benefits compared to the same attribute values on other ship.

\section{Fishing equipment viewed from social aspect}

The factors used in the social aspects are the number of manpower (ABK), income of fishermen per year (Rp), experience of fishermen, the possibility of ownership, operational security. Based on the results of the scoring, the fishing equipment of the redi HU opened up employment because it requires a greater number of crew members compared to other fishing gear. Besides that H.U's fishing treatment can provide higher profits, so that manpower's income was also high. According to Monintja (1987) attemps to develop one type of fishing treatment in one region, the thing that must be considered is that it can absorb more labor. Manpower (ABK) can absorb technology and its update easily in the operation 
of fishing equipment. According to Nurhakim and Merta (2004), that fisheries management must pay attention to environmental factors (ecosystems). Furthermore, it is said that management can be achieved with a conservation pattern between resource use which can still preserve the aquatic environment as a target fishing habitat.

\section{Combination Aspect}

Based on the results of standardization of combined aspects assessment, namely the biological, technical, social, economic and ecosystem aspects, the priority for development in Buton District was H.U's fishing equipment (table 12 and table 13). This combination analysis aimed to see and assess the appearance of the fishing gear used by fishermen as a whole and serve as an indicator in the development and sustainability of redi fishingequipment in the Tengon Buton Regency of Sulawesi. From the results of the joint assessment above, it is clear that the H.U redi fishing equipment can be developed for the utilization of Buton district pelagic fish resource. Considering that purse seine is a redi fishing tool that can be developed by Buton Regency fishermen, the regulation of the amount and capacity that must be fulfilled is highly dependent on the regulation of policies by the local government. Capture capacity is defined as the ability of fishing ship units with all equipment owned and reviewed from all aspects to carry out fishing (Musyafak et al 2009). According to Wiyono (2011), the use of fishing equipment must refer to the potential resources available in one region. Considering the potential of redi fisheries in Buton District is getting thinner and very vulnerable to changes in oceanographic factors globally, it is necessary to immediately adjust the number of operated fishing gear, so that the availability of resources in their habitat can be maintained.

\section{CONCLUSION}

Based on the results of research of appropriate types selection of redi fishing equipment and appropriate technology in the utilization of small pelagic fish resources with redi fishing equipment, it can be concluded that H.Usman redi fishing equipment fulfilled the criteria as a sustainable fishing gear in the Buton District, Southeast Sulawesi

\section{SUGGESTION}

The next research should be focused on the study of sustainability dimension attributes by taking into account the mastery of thestudy field, especially the interaction with other scientific fields.

\section{REFERENCES}

- Amin et al. 2000. Pengaruh Intensitas Cahaya dan Ukuran Mata Jaring Terhadap Selektivitas Payang Oras Bagi Penangkapan Cumi. Di dalam : Suparno,editor. Prosiding Seminar Hasil Penelitian Perikanan; Sukamandi, 2122 September 2000. Jakarta : Pusat Penelitian dan Pengembangan Eksplorasi Laut dan Perikanan. Hal 125-132.

- Aminah, S. 2009. Analisis Pemanfaatan Sumberdaya Ikan Kembung (Rastrelliger spp) di Perairan Kabupaten Tanah Laut Provinsi Kalimantan Selatan. Thesis. Tidak dipublikasikan. Sekolah Pascasarjana Institut Pertanian Bogor. 64 hal.

- Ayodyoa. 1984. Metode Penangkapan Ikan, Fakultas Perikanan. Institut Pertanian Bogor. Yayasan Dewi Sri.

- Brant, A.V. 1984. Fish captureing Methods of the world. England: Fishing News Books. 418 p

- Chan, M.S. 1997. The Global Burden of Intestinal Nematode Infections: Fifty Years on. Parasitol Today, 13:43843.

- Dahuri. 2007. Membenahi Sistem Perikanan Tangkap. http://dahuri.wordpress.com

- Dinas Kelautan dan Perikanan Provinsi Jawa Timur. 2011. Laporan Statistik Perikanan tangkap tahun 2011

- Dinas Kelautan dan Perikanan Provinsi Bali. 2011. Laporan Statistik Perikanan tangkap tahun 2011

- [FAO] 1995. Food and Agreculture Organization of The United Nations. Code of Conduct for Responsible Fisheries. Rome. 45p.

- Fauzi A, Anna S. 2005. Pemodelan Sumberdaya Perikanan dan Kelautan, Untuk analisis Kebijakan. Penerbit PT. Gramedia Pustaka Utama. Jakarta. 399 hal.

- Haluan J, TW Nurani. 1988. Penerapan Metode Skoring dalam Penelitian Teknologi Penangkapan Ikan yang Sesuai untuk Dikembangkan di Suatu Wilayah Perairan. Buletin PSP Vo. II No. 1. Fakultas Perikanan. Institut Pertanian Bogor. Hal. 3-16.

- Lee, J. W. 2010. Pengaruh Periode Hari Bulan terhadap Hasil Tangkapan dan Tingkat Pendapatan Nelayan Bagan Tancap di Kabupaten Serang. Thesis. Tidak dipublikasikan. Sekolah Pascasarjana Institut Pertanian Bogor. 99 Hal.

- Mangkusubroto, K dan Trisnadi. 1985. Analisa Keputusan Pendekatan Sistem dalam Manajemen Usaha dan Proyek. Penerbit Ganeca Exact. Bandung. 271 hal.

- Merta I G S. 1992. Dinamika Populasi Ikan Lemuru Sardinella lemuru Bleeker 1853 (pisces: Clupeidae) di Perairan Selat Bali dan Alternatif Pengelolaannya. Sekolah Pasca Sarjana Institut Pertanian Bogor. 228 hal.

- Monintja D.R. 1987. Beberapa Teknologi Pilihan untuk Pemanfaatan Sumberdaya Hayati Laut di Indonesia. Buletin PSP Vol. 1 No. 1. Fakultas Perikanan. Institut Pertanian Bogor. Hal. 14-25. 
- Musyafak, A Rosyid, A Suherman. 2009. Kapasitas Penangkapan Kapal Pukat Cincin di Pelabuhan Perikanan Nusantara Pekalongan. Jurnal Saintek Perikanan. Vol. 4 No. 2. Universitas Diponegoro. Hal. 16-23

- Nontji, A. 2007. Laut Nusantara. Edisi revisi. Penerbit Djambatan. 367 hal

- Nurhakim S dan Merta. 2004. Perkembangan dan pengelolaan perikanan lemuru, Sardinella lemuru Bleeker 1853 di Selat bali. Jurnal Penelitian Perikanan Indonesia. Vol. 10 No. 4. Edisi Sumberdaya dan Penangkapan Tahun 2004. Badan Riset Kelautan dan perikanan Departemen Kelautan dan Perikanan. Hal. 53-63.

- Sudirman. 2003. Analisis Tingkah Laku Ikan untuk Mewujudkan Teknologi Ramah Lingkungan dalam Proses Penangkapan pada Bagan Rambo. Disertasi. Tidak Dipublikasikan. Sekolah Pascasarjana Institut Pertanian Bogor. $231 \mathrm{Hal}$.

- Widodo J. 2000. Model-Model Surplus Produksi untuk Mengestimasi Hasil Tangkapan Maksimum Lestari (Maximum Sustainable Yield-MSY). Penuntun Pengkajian Stok Sumberdaya Ikan Perairan Indonesia BRKPDKP dan P2O-LIPI. Jakarta. p 49-60

- Wiyono, E.S. 2011. Alat Tangkap Unggulan di Kabupaten Bangka Selatan, Provinsi Bangka Belitung. Buletin PSP. Volume XIX No. 3 Edisi Desember 2011. Institut Pertanian Bogor. Hal 229-238. 